\title{
Brownian motors: current fluctuations and rectification efficiency
}

\author{
L. Machura, ${ }^{1,2}$ M. Kostur, ${ }^{1}$ P. Talkner,${ }^{1}$ J. Euczka, ${ }^{2}$ F. Marchesoni, ${ }^{3}$ and P. Hänggi ${ }^{1}$ \\ ${ }^{1}$ Institute of Physics, University of Augsburg, Universitätsstrasse 1, D-86135 Augsburg, Germany \\ ${ }^{2}$ Institute of Physics, University of Silesia, P-40-007 Katowice, Poland \\ ${ }^{3}$ Dipartimento di Fisica, Università di Camerino, I-62032 Camerino, Italy
}

(Dated: September 5, 2006)

\begin{abstract}
With this work we investigate an often neglected aspect of Brownian motor transport: The rôle of fluctuations of the noise-induced current and its consequences for the efficiency of rectifying noise. In doing so, we consider a Brownian inertial motor that is driven by an unbiased monochromatic, time-periodic force and thermal noise. Typically, we find that the asymptotic, time- and noiseaveraged transport velocities are small, possessing rather broad velocity fluctuations. This implies a corresponding poor performance for the rectification power. However, for tailored profiles of the ratchet potential and appropriate drive parameters, we can identify a drastic enhancement of the rectification efficiency. This regime is marked by persistent, uni-directional motion of the Brownian motor with few back-turns, only. The corresponding asymmetric velocity distribution is then rather narrow, with a support that predominantly favors only one sign for the velocity.
\end{abstract}

PACS numbers: 05.60.Cd, 05.40.-a, 05.45.-a

\section{INTRODUCTION}

The channelling of particles by harvesting the thermal noise gives rise to a diffusive transport of particles. In periodic potentials the second law of thermodynamics implies that no net transport occurs. The situation changes drastically, however, in the presence of unbiased non-equilibrium noise which acts additionally on such systems. Then, the concept of Brownian motors [1] does provide the possibility for directed, noise-induced transport. The phenomenon has widespread applications in physics, chemistry and in the biological sciences where it can be put to work for shuttling reliably and efficiently particles on the micro-scale, or even on the nano-scale 1].

The vast majority of works on Brownian motors concentrate on the behavior and the selective control of the emerging directed transport as a function of control parameters such as the temperature $T$, an external load $F$ (yielding the load-current characteristics), or some other control variable. In contrast, the role of the fluctuations of the directed current has not attracted much attention in the literature. A notable exception is the first work on an inertial (rocking) ratchet 2] wherein the higher order, statistical cumulant properties of the stochastic position variable have been explored. Here, we fill this gap and focus in more detail on the fluctuating behavior of the Brownian motor current. The average drift motion together with its fluctuation statistics are salient features when characterizing the modus operandi of a particular Brownian motor.

It is intuitive that the fluctuations of the drift variable do impact the overall "efficiency" of the transport under consideration. The objective of an optimal operation of a Brownian motor machine can be formulated in a variety of ways, e.g. see in 3]. In close analogy to heat-engine machines, one can define a generalized, nonequilibrium efficiency of a Brownian motor. There exists no univer- sally agreed upon definition of this notion [4, 5, 6, 7] for a recent review on efficiency of Brownian motors see Ref. [8]. The most common definition of the efficiency of a Brownian motor operating at non-equilibrium is based on the ratio of the work (or power) done by the particle against an external load and the input energy (input power). With this working definition the load force is inevitably included; in particular, this yields the result that the efficiency assumes a zero value when no load force is acting.

Alternative proposals for efficiencies have been proposed as well [5, 6, 7]: Some of these proposals do provide a nonzero value for a vanishing bias force. Yet another possibility consists in characterizing the rectification power of the transport in terms of the so-called Peclet number, i.e. the quotient of the drift velocity and the diffusion. This notion has been used in continuous state Brownian motor transport 9], and also for discrete motor models 11]. The concept is related in spirit to a Fano factor measure [12] of the velocity fluctuations used, for example, to characterize molecular motors 13].

In this work, we shall follow the reasoning of Suzuki and Munakata [7], in order to characterize the efficiency of rectification in absence of external bias forces. Typically, there occurs a competition between two mechanisms: a "giant enhancement" of diffusion 9, 10, 14, 15] and an optimally large, (uni)-directional transport velocity [16, 17]. The first perspective aims at controlling the magnitude of the effective diffusion independently of the temperature. It thus carries a rich potential for technological separation devices. The second facet attempts to achieve a maximal "coherence" for the transport. Such a coherence is of relevance for Brownian motors modelling biophysical molecular motors [18].

Most of the Brownian motors and the majority of applications studied in the prior literature operate in the socalled overdamped Brownian motion regime. For specific applications the rôle of inertial effects can become, how- 
ever, of salient importance 2, 10, 19, 20, 21, 22, 23, 24, $25,26,27,28]$. The overdamped dynamics is a valid approximation for many physical applications [1]. It is also particularly well suited to describe the motion of molecular motors 1, 18]. In other situations, however, the inertial effects cannot be neglected. An exemplar is the diffusion of atoms on a crystal surface 29]. There, the dynamics may be underdamped, exhibiting long correlated hopping among binding sites. This physics has been verified experimentally by use of scanning tunnelling microscopy [30], field ion microscopy [31], or for quasi-elastic Helium atom scattering [32].

The inclusion of inertia adds significant complexity to the problem. This is so, because a periodically rocked, single degree of freedom with nonzero mass possesses a three-dimensional phase space that can exhibit a chaotic dynamics 2, 20, 21]. This is in contrast to the case of rocked, overdamped Brownian motors 33, 34, 35, 36, 37]. While the chaotic dynamics of a driven-damped particle in a symmetric periodic potential has been investigated thoroughly during the 1980's, the (chaos)-induced, directed transport of an asymmetric, inertial Brownian motor has been pioneered only much later in [2]. There, it has been demonstrated that the corresponding dynamics features a rich structure, possessing many intriguing current-reversals. In the deterministic case, different asymptotic solutions can coexist, e.g. running and locked states. Moreover, the onset of diffusive behavior due to chaotic dynamics has been investigated in terms of the second moment of the particle position diffusion. This first study was followed up with more detailed investigations [20], where it has been shown, for example, that the transport may reverse the direction at the transition from a chaotic to a regular motion. Additionally, intermittent trajectories have been observed. In such cases the system follows for a certain time a regular orbit, but then suddenly switches to a sticking orbit. The resulting average flux depends on the time which the particle spends in a particular state, and the system may exhibit super-diffusive behavior. In related work 24], the inertial Brownian motor dynamics in a regime of adiabatic driving has been investigated. These authors focused on the onset of the diffusive transport as the damping coefficient decreases.

In this work we concentrate on the connection between the directed transport and its fluctuation characteristics. This study is of relevance for the optimization of rectification: The directed current should not become swamped with the unavoidable fluctuations of the transported quantity. Our investigation is based on an inertial, noise-driven rocking ratchet. We shall analyze quantities such as: the long-time averaged velocity, its velocity fluctuations, the fluctuations of the kinetic energy of the Brownian motor and the efficiency for rectification.

The paper is organized as follows. In the next section we present the Brownian motor model. In Section III, we elaborate on the problem of the efficiency for rectifying noise in connection with the fluctuation behavior of the
Brownian motor current. In Section IV, we describe our numerical findings for a generic set of parameters, while in Section V, we elucidate the optimal working conditions for rectification and directed transport.

\section{MODEL}

To start, we consider the motion of a classical particle of mass $m$ moving in the periodic, asymmetric ratchet potential $V(x)$. The particle is driven by an unbiased time-periodic, monochromatic force of strength $A$ and an angular frequency $\Omega$. The dynamics is additionally subjected to thermal noise. The Brownian motor dynamics is thus governed by the Langevin equation [39]

$$
m \ddot{x}+\gamma \dot{x}=-V^{\prime}(x)+A \cos (\Omega t)+\sqrt{2 \gamma k T} \xi(t),
$$

where the prime denotes a differentiation with respect to the argument of $V(x)$. The parameter $\gamma$ is the friction coefficient, $T$ denotes temperature, and $k$ is the Boltzmann constant. The ratchet potential $V(x)=V(x+L)$ has the period $L$ and a barrier height $\Delta V$. Thermal fluctuations are modelled by the zero-mean $\delta$-correlated Gaussian white noise $\xi(t)$. This noise term obeys the Einstein relation with the noise correlation given by $\langle\xi(t) \xi(s)\rangle=\delta(t-s)$. We next introduce dimensionless variables. The natural length scale is given by the period $L$ of the ratchet potential. The dynamics possesses several time scales. We introduce the characteristic time $\tau_{0}$ as determined formally from the Newton equation, $m \ddot{x}=-V^{\prime}(x)$, by balancing the two forces $m L / \tau_{0}^{2}=\Delta V / L ;$ yielding $\tau_{0}^{2}=m L^{2} / \Delta V$. The scaled variables thus read:

$$
\hat{x}=\frac{x}{L}, \quad \hat{t}=\frac{t}{\tau_{0}} .
$$

The dimensionless Langevin dynamics consequently assumes the form

$$
\ddot{\hat{x}}+\hat{\gamma} \dot{\hat{x}}=-\hat{V}^{\prime}(\hat{x})+a \cos (\omega \hat{t})+\sqrt{2 \hat{\gamma} D_{0}} \hat{\xi}(\hat{t}),
$$

where

- the re-scaled friction coefficient $\hat{\gamma}=(\gamma / m) \tau_{0}$ is the ratio of the two characteristic time scales, $\tau_{0}$ and the relaxation time scale of the velocity degree of freedom, i.e., $\tau_{L}=m / \gamma$,

- the potential $\hat{V}(\hat{x})=V(x) / \Delta V$ assumes the period 1, and the barrier height equals unity; i.e. $\Delta \hat{V}=1$,

- the drive has the re-scaled force strength $a=$ $A L / \Delta V$ with the dimensionless angular frequency $\omega=\Omega \tau_{0}$,

- the re-scaled, zero-mean Gaussian white noise forces $\hat{\xi}(\hat{t})$ obey $\langle\hat{\xi}(\hat{t}) \hat{\xi}(\hat{s})\rangle=\delta(\hat{t}-\hat{s})$ with a rescaled noise intensity $D_{0}=k T / \Delta V$. 
In the following, mostly for the sake of simplicity, we shall only use dimensionless variables and shall omit the "hat"notation in all quantities. For the asymmetric ratchet potential $V(x)$ we consider a linear superposition of at least two (or even three) spatial harmonics (see in Sect. IV), i.e.,

$$
V(x)=V_{0}\left[\sin (2 \pi x)+c_{1} \sin (4 \pi x)+c_{2} \sin (6 \pi x)\right],
$$

wherein $V_{0}$ normalizes the barrier height to unity, and the parameters $c_{1}$ and $c_{2}$ characterize the spatial asymmetry.

\section{FLUCTUATION AND RECTIFICATION MEASURES}

Throughout the following we focus on the asymptotic, periodic regime after effects due to the influence of initial conditions and transient processes have quiet down. Then, the main statistical quantifiers of the driven stochastic process can be described in terms of timeand ensemble-averages. For a given quantity $f(x(t))$ its time-homogeneous statistical properties are obtained only in the long-time limit after transients have died out and after both, the average over the temporal period of the driving and the corresponding ensemble-average are performed 38]. In this asymptotic regime the timeindependent (single-time) quantities are obtained by a double averaging procedure over both the noise and the period of driving; i.e.,

$$
\langle f\rangle=\lim _{t \rightarrow \infty} \frac{\omega}{2 \pi} \int_{t}^{t+2 \pi / \omega} \prec f(x(s)) \succ d s,
$$

wherein $\prec f(s) \succ$ indicates the average over the noise realizations (ensemble-average).

The most salient transport quantity is the average, directed velocity $\langle v\rangle$ of the driven Brownian particle. Here $v(t)$ denotes the stochastic process $\dot{x}(t)$ in Eq. 3. Of equal importance are, however, the fluctuations of $v(t)$ around its mean $\langle v\rangle$ in the long time regime, i.e., the variance

$$
\sigma_{v}^{2}=\left\langle v^{2}\right\rangle-\langle v\rangle^{2}
$$

The Brownian motor moves with current values $v(t)$ that range typically within

$$
v(t) \in\left[\langle v\rangle-\sigma_{v},\langle v\rangle+\sigma_{v}\right] .
$$

If $\sigma_{v}>\langle v\rangle$, and even more so if $\sigma_{v} \gg\langle v\rangle$, the Brownian motor can possibly move for some time in the opposite direction of its average value $\langle v\rangle$. The question thus arises: Is an efficient directed transport still feasible?

To answer this challenge we shall introduce a measure for the efficiency $\eta$ of the rectification of thermal noise, a quantity directly related to the velocity fluctuations. Here, we follow the reasoning of Suzuki and Munakata
7], which yields a nonvanishing rectification efficiency also in the absence of an external bias. This efficiency of rectification follows from an energy balance of the underlying inertial Langevin dynamics. When specialized to our situation, $\eta$ is given by the ratio of the dissipated power $\gamma\langle v\rangle^{2}$ associated with the directed motion of the motor against friction, and the input power from the time-periodic forcing. The result assumes the explicit form (see in the Appendix)

$$
\begin{aligned}
\eta & =\frac{\langle v\rangle^{2}}{\left|\langle v\rangle^{2}+\sigma_{v}^{2}-D_{0}\right|}, \\
& =\frac{\langle v\rangle^{2}}{\left|\left\langle v^{2}\right\rangle-D_{0}\right|} .
\end{aligned}
$$

It thus follows that for a decreasing variance of the velocity fluctuations, $\sigma_{v}^{2}$, the efficiency of the Brownian motor increases. This is just what one would expect on naive grounds: The transport of a Brownian motor can be optimized in regimes of a large, directed average current which intrinsically does exhibit only small fluctuations. Moreover, in our study we found numerically that $\left\langle v^{2}\right\rangle>D_{0}$ holds true for any chosen set of the simulation parameters.

\section{FLUCTUATION BEHAVIOR OF CURRENT IN AN INERTIAL ROCKED BROWNIAN MOTOR}

Deterministic inertial Brownian motors exhibit a complex dynamics including chaotic regimes. The application of noise then generally destroys the complex fine structure of their phase space and tends to smooth out their characteristic response function.

There are two classes of states of the driven system dynamics: the locked states, in which the particle stays inside one potential well, and the running states for which the particle runs over the potential barriers. The first regime is characteristic for small driving strengths. When the amplitude of the external force is made sufficiently large we find that running states appear. These running states can either be chaotic (diffusive) or regular.

\section{A. Numerical schemes}

We have carried out extensive numerical studies in order to identify generic properties of the noise-induced transport. Applying a small, but finite noise strength, $D_{0}>0$, we have integrated the Langevin equation (3) by employing the Euler method with a time step of $h=10^{-3}$. For the initial condition of the coordinate $x(t)$ we used a uniform distribution over the dimensionless period $L=1$ of the ratchet potential. Likewise, the (scaled) starting velocity has been chosen at random from a symmetric, uniform distribution over the interval $[-1,1]$. All quantities of interest were averaged over 250 
different trajectories. Each single trajectory evolved over $45 \times 10^{3}$ periods. The transient regime usually relaxed already long before 500 periods of the driven dynamics had elapsed. For the cases of very weak noise and weak driving we extended the corresponding time-span to ensure that the transient dynamics has quiet down completely. In the limiting deterministic case, i.e. $D_{0}=0$, we used the Runge-Kutta algorithm of order 5 . In this case, the averages were calculated over $10^{3}$ differing trajectories, each trajectory evolving over $10^{3}$ periods.

\section{B. Numerical results: Current fluctuations versus driving strength}

We start out to study the role of fluctuations by varying the amplitude $a$ of the sinusoidal driving force. In doing so, we assume a relatively small temperature, so that the Brownian motor dynamics is not far from a deterministic behavior as described in prior works 2, 20, 24]. The average asymptotic long-time current velocity is shown in Fig. 1(a). It reveals that for an amplitude $a \simeq 1.5$ the directed, inertial transport sets in before the lower threshold of the ratchet force is reached. It assumes a first local maximum near the lower threshold of the potential force near $a \simeq 2.1$. Note that in the presence of small noise, the current is strictly speaking never zero. For all practical purposes, however, we can characterize the outcome of our Langevin simulation as a deterministic, zero-current result. Below this threshold the system mainly dwells in the locked state. Upon closer inspection, we notice that in the vicinity of $a \simeq 0.6$, the velocity fluctuations $\sigma_{v}$ shown in Fig. 1(b) undergo a rapid increase. In Fig. 11 c) we also display the relative fluctuations of the kinetic energy, $E=v^{2} / 2$, (the re-scaled mass is one); i.e.

$$
\frac{\sigma_{E}^{2}}{\langle E\rangle^{2}}=\frac{\left\langle v^{4}\right\rangle-\left\langle v^{2}\right\rangle^{2}}{\left\langle v^{2}\right\rangle^{2}}
$$

Around this value of the driving amplitude, such a quantity undergoes a giant enhancement. Finally, we remark that for the equilibrium Maxwell distribution we find $\sigma_{E}^{2} /\langle E\rangle^{2}=2$, as expected.

Upon further increasing the amplitude of driving, $a>$ 1.5, the Brownian motor generates for this set of parameters the desired, directed transport behavior. At the same time we observe that the width of the weakly asymmetric, corresponding distribution $P(v)$ slightly decreases, meaning that the velocity fluctuations become smaller. The following explanation thus applies: Because at $a<1.5$ escape jumps between the neighboring wells are rare, i.e., the average directed current is very small (note also the accompanying, very weak asymmetry in the velocity distribution). The input energy is pumped primarily into the kinetic energy of the intra-well motion and eventually dissipated. As $a$ is increased further, the Brownian motor mechanism starts to work, and some

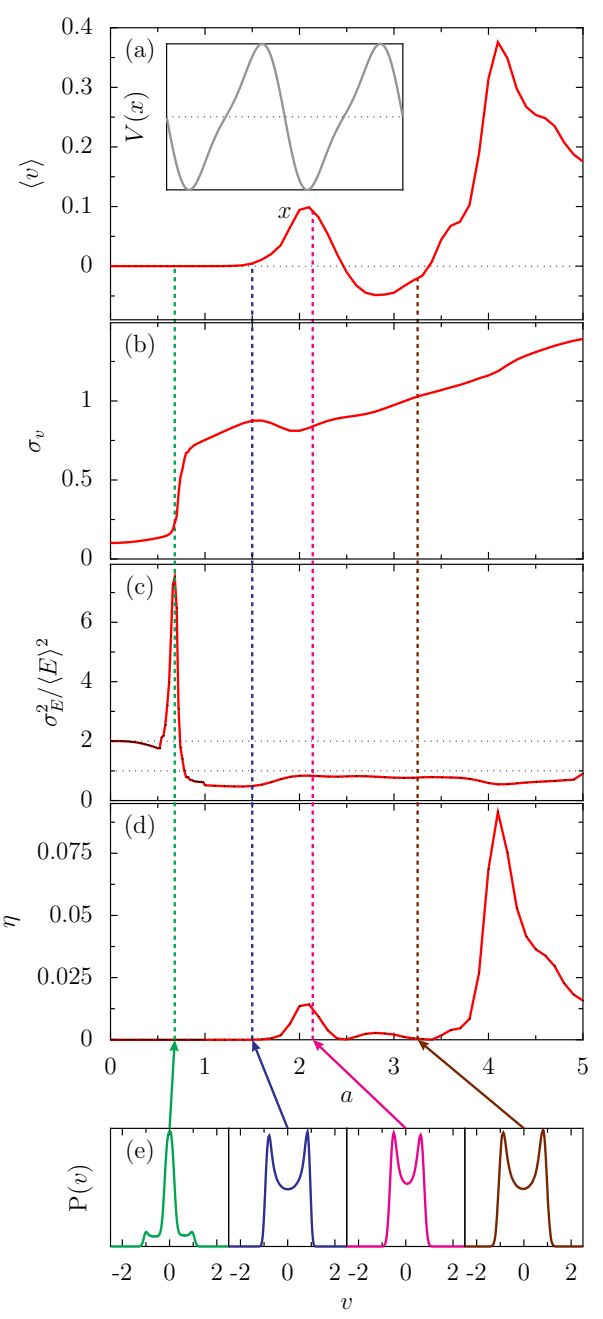

FIG. 1: (Color online) Fluctuation behavior of an inertial Brownian motor versus the driving strength $a$. (a): averaged dimensionless velocity $\langle v\rangle$ of the inertial Brownian motor in Eq. (3); (b): variance of the corresponding velocity fluctuations $\sigma_{v}$; (c): fluctuations of the rescaled kinetic energy $\sigma_{E}^{2} /\langle E\rangle^{2} ;(\mathrm{d})$ : rectification efficiency in Eq. (8). All quantities have been computed for the rescaled potential $V(x)=-V_{0}[\sin (2 \pi x)+0.25 \sin (4 \pi x)]$, where $V_{0} \simeq 0.454$ normalizes the barrier height to unity, see the inset in (a). The force corresponding to this potential ranges from -2.14 to 4.28. The angular frequencies at the well-bottom and at the barrier-top, respectively, equal each other, reading 5.28. Bottom panel (e): velocity distributions for selected driving amplitudes, i.e. $a=0.68,1.5,2.14,3.25$. All these distributions were normalized by setting their maximum to one. The remaining rescaled parameters read: friction $\gamma=0.5$, angular driving frequency $\omega=3.6$ and weak thermal noise of strength $D_{0}=0.01$.

part of energy contributes to the net motion of the particle. Therefore, less energy remains available to drive intra-well oscillations and consequently the distribution $P(v)$ shrinks, see in Fig. 11(e)

Correspondingly, due to inertia, the mean velocity increases, reaching a second maximum before the upper 
threshold value of the potential force $a \simeq 4.28$. Above this driving amplitude, the current starts to decrease because of the weakening influence of the ratchet potential at large rocking amplitudes.

The occurrence of multiple reversals for the directed current, as it occurs in Fig. 1(a), is a known, interesting feature of inertial Brownian motors. Several prior studies did elucidate in greater detail the corresponding mechanism at work 2, 20, 22, 24, 25]. Here, we take instead a closer look at the current fluctuations. We observe that for the chosen set of parameters the maximal stationary velocity in Fig. 1(a) does not exceed the value 0.4. In contrast, its fluctuations keep growing as the driving amplitude rises. At large driving, the particle no longer feels the potential and undergoes a rocked, free Brownian motion with the velocity fluctuations growing proportional to $a$, cf. Fig. 1(b).

On the other hand, the relative fluctuations of the kinetic energy do saturate, see Fig. 11(c). These are suppressed to values near 1 , which lies below the equilibrium value of 2 . Within this directed transport regime, the efficiency (8) remains rather small, cf Fig. 1(d). Such small rectification efficiency is the rule for this driven inertial Brownian motor.

Let us next inspect the current probability distribution $P(v)$. All $P(v)$ curves reported in the following have been normalized so that their maximum (i.e., their highest peak) is set to a fixed, unit value. Only then we can detect the details in their shape upon varying the corresponding parameter such as the driving amplitude $a$ or the noise strength $D_{0}$. These probabilities look rather symmetric; however, a finite ratchet velocity requires a certain amount of asymmetry either in the location and/or the width of the velocity peaks. Here, the current results mainly due to a slight shift of the maxima location.

The most peculiar feature of the current distributions shown in Fig. 1(e) is the emergence for $a>0.6$ of two additional side-peaks centered near $v= \pm 1$, which eventually dominate $P(v)$ at larger driving amplitudes. Of course, for zero drive $P(v)$ boils down to a single-peaked Maxwell distribution, strictly symmetric around $v=0$. To investigate the onset of these two side-peaks for $a$ values corresponding to vanishingly small currents, we set $P(v)=q P_{0}(v-1)+q P_{0}(v+1)+(1-2 q) P_{0}(v)$, where $q$ varies from 0 to 1 . If $P_{0}$ is taken to be a symmetric Gaussian function, then the kinetic energy fluctuations can be evaluated; $\sigma_{E}$ exhibits the behavior shown in Fig. $1(\mathrm{c})$.

What it the origin of those three peaks in the distribution $P(v)$ ? Our first conjecture to connect it with the 'running' solutions turned out to be incorrect. This is so, because for $a \lesssim 1$ the particle rarely leaves the confining potential well and thus cannot significantly contribute to the side peaks of the distribution function. We further checked the outcome for the velocity distribution when reflecting barriers were placed at the maxima of the potential. Under such constraints, the three-peak- structure is recovered as well. Moreover, the sinusoidally driven damped particle in a harmonic potential can exhibit both, a singly-peaked as well a doubly-peaked averaged velocity distribution, see in Ref. 38]. However, for the parabolic potential that fits best the wells of our ratchet potential around its minima, we found a single peaked $P(v)$.

We therefore do conclude that the characteristic behavior for the additional side-peaks is rooted in the nonlinear, anharmonic character of the corresponding well of the periodic asymmetric ratchet profile.

\section{Numerical results: role of the shape of the underlying ratchet potential}

We next study the influence of the ratchet profile (or force) for the mean velocity and the corresponding fluctuation behavior of the directed current. We use a stylized potential shape composed of three spatial higher harmonics with $c_{2} \neq 0$, see in (4). Note that this potential shape possesses an opposite polarity as compared with the ratchet potential depicted in the inset of Fig. 1(a). Put differently, the natural direction of the Brownian motor motion is to the left, in the direction of the weaker slope, see Fig. 2(a), inset.

For small driving amplitudes, this inertial motor predominantly dwells in a potential well. The directed current is very small and negative (directed towards the left). If the driving amplitude exceeds the upper threshold amplitude of the ratchet force at $a \simeq 4.66$, the motor starts to move more regularly, reaching an extremal speed near $a \simeq 6$. Interestingly enough, the motor moves now towards positive $x$-values. The resulting transport velocity thus cannot be easily predicted a priori in this nonadiabatic driving regime. This is a benchmark feature of these inertial Brownian motors where the coupling between the deterministic driving force, $F(t)=a \cos (\omega t)$, and the resulting motion of the driven Brownian particle are coupled loosely, only. Except for a narrow regime of sizable values, cf. Fig. 2(a), the emerging average velocities are typically very small, yielding no corresponding efficient rectification. Therefore, the efficiency of this more complex Brownian motor mimics again closely the behavior of the average motor velocity, see panel (d) in Fig. [2]

The velocity fluctuations exhibit a similar behavior as in the case of Fig. 1 discussed above. The variance grows nearly linearly with increasing driving strength. Typically, the average velocities are small and the fluctuation behavior is similar to the behavior discussed above for the first ratchet potential. Interestingly enough, however, above threshold driving induces a distinct peak behavior for the velocity which is accompanied by a corresponding dip in the behavior for the velocity fluctuations. This dip in the variance then gives rise to a window with appreciable efficiency, cf. Fig. 2(d).

The behavior for the velocity distributions is again 


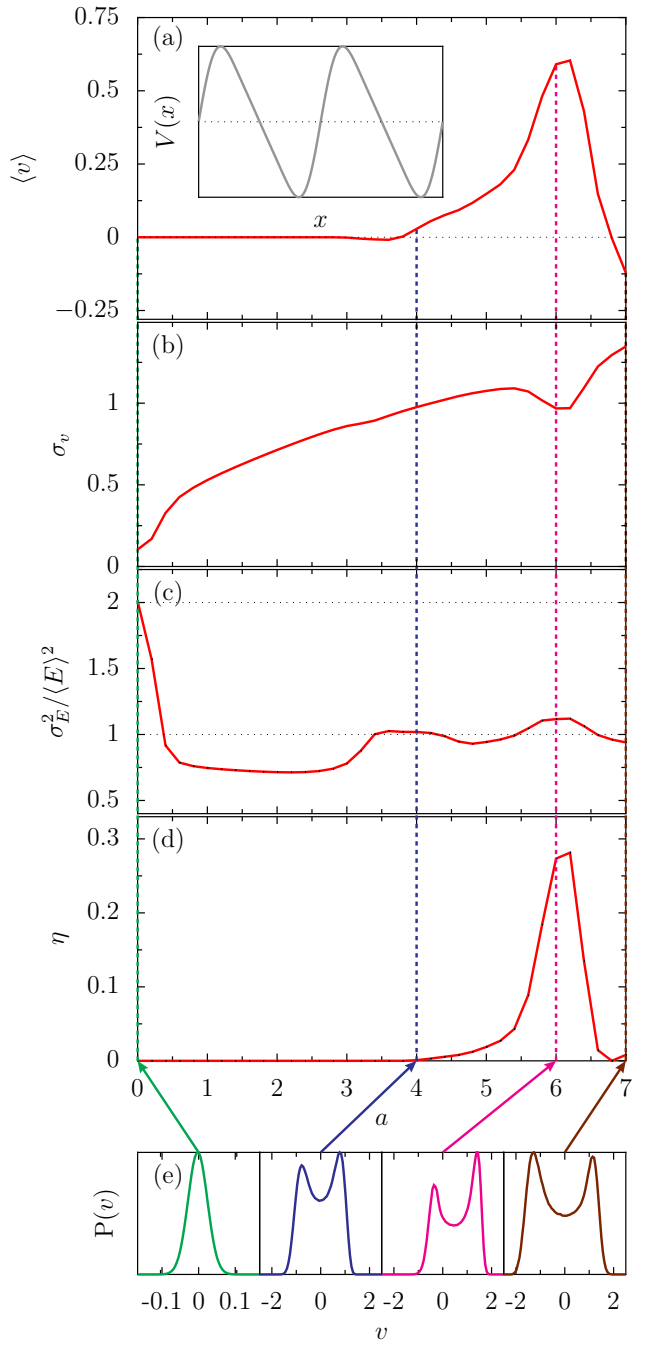

FIG. 2: (Color online) Tailoring the shape of the potential. (a): averaged dimensionless velocity $\langle v\rangle$ of the inertial Brownian motor under nonadiabatic driving conditions; (b): corresponding velocity fluctuations $\sigma_{v}$; (c): fluctuations of the rescaled kinetic energy $\sigma_{E}^{2} /\langle E\rangle^{2}$; (d): efficiency. All quantities are plotted versus the external driving amplitude $a$ for the asymmetric ratchet potential $V(x)=V_{0}[\sin (2 \pi x)+$ $0.245 \sin (4 \pi x)+0.04 \sin (6 \pi x)]$, where $V_{0} \simeq 0.461$ normalizes the barrier height to unity (see inset in (a)). The forces stemming from such a potential range between -4.67 and 1.83 . The two angular frequencies at the well-bottom and at the barrier-top are the same, reading 5.34. The corresponding velocity distributions $P(v)$ are displayed in panel (e) for the indicated driving amplitudes, i.e., $a=0,4,6,7$. All $P(v)$ curves have been normalized by setting their maximum to one. The remaining parameters are: $\gamma=0.9, \omega=4.9$ and $D_{0}=0.01$.

generic: Small average velocities exhibit nearly symmetric velocity distributions, see Fig. 2(e). Only for the nonadiabatic peak behavior of the mean velocity does one identify also an appreciable asymmetry for the velocity distribution.

\section{Numerical results: Current fluctuations versus noise strength $D_{0}$}

In Fig. 3, we numerically investigate the directed transport versus the temperature $D_{0}$. We have chosen a sub-threshold driving strength for which the thermal noise plays a constructive role [40] by inducing noise activated jumps across the potential barriers. We set $a=0.8$ and the other parameters remain the same as in Fig. 11 Then, we find a characteristic velocity reversal near the dimensionless temperature $D_{0}=k T / \Delta V \simeq 1$. There, the thermal energy compares with the activation energy over the barrier height of the ratchet potential. A subsequent increase of temperature causes a diminishing role of the asymmetric ratchet potential and, consequently, the directed transport degrades.

Moreover, the time-averaged velocity distribution approaches the equilibrium velocity distribution [9]. It is remarkable that within a certain range of temperatures the fluctuations of the kinetic energy exceed the relevant value for the Maxwellian equilibrium distribution, cf. Fig. 31(c). A shallow, local minimum occurs for the velocity fluctuations where the average current itself is maximal. These fluctuations are, however, notably three orders of magnitude larger than the small-valued, directed current. Not surprisingly, the rectification efficiency shown in Fig. 3(d) is quite small. Again, the Brownian motor is not operating optimally.

\section{TAILORING RECTIFICATION EFFICIENCY}

Thus far, changing the ratchet profiles did not lead to a large enhancement of the rectification efficiency. What is needed in achieving a large rectification efficiency is a sizable Brownian motor current which is accompanied by small current fluctuations only, see Eq. (8). This scenario seemingly implies that the directed current should proceed in a persistent manner with very few, occasional back-turns only. This in turn causes small fluctuations in the velocity and, additionally, provides a dominating asymmetry of the velocity distribution.

Such a behavior can be realized by a combined tailoring of the asymmetry of the ratchet potential together with the use of appropriate driving conditions. In the quest for achieving such a favorable situation we use the three-harmonics ratchet potential plotted in the inset of Fig. 2(a). Our hope is that upon minimizing the noise further we can achieve a substantial improvement of the efficiency.

At very weak noise and large, nonadiabatic rocking frequencies, this inertial Brownian motor starts moving efficiently near the upper threshold of the ratchet force $a \simeq 4.66$, see Fig 4 (a). Because the directed velocity becomes maximal and simultaneously its fluctuations are locally minimal, see in Fig. 4(b), we indeed find the desired enhancement of the rectification efficiency, see Fig. 4(d). The fluctuations of the kinetic energy grow 


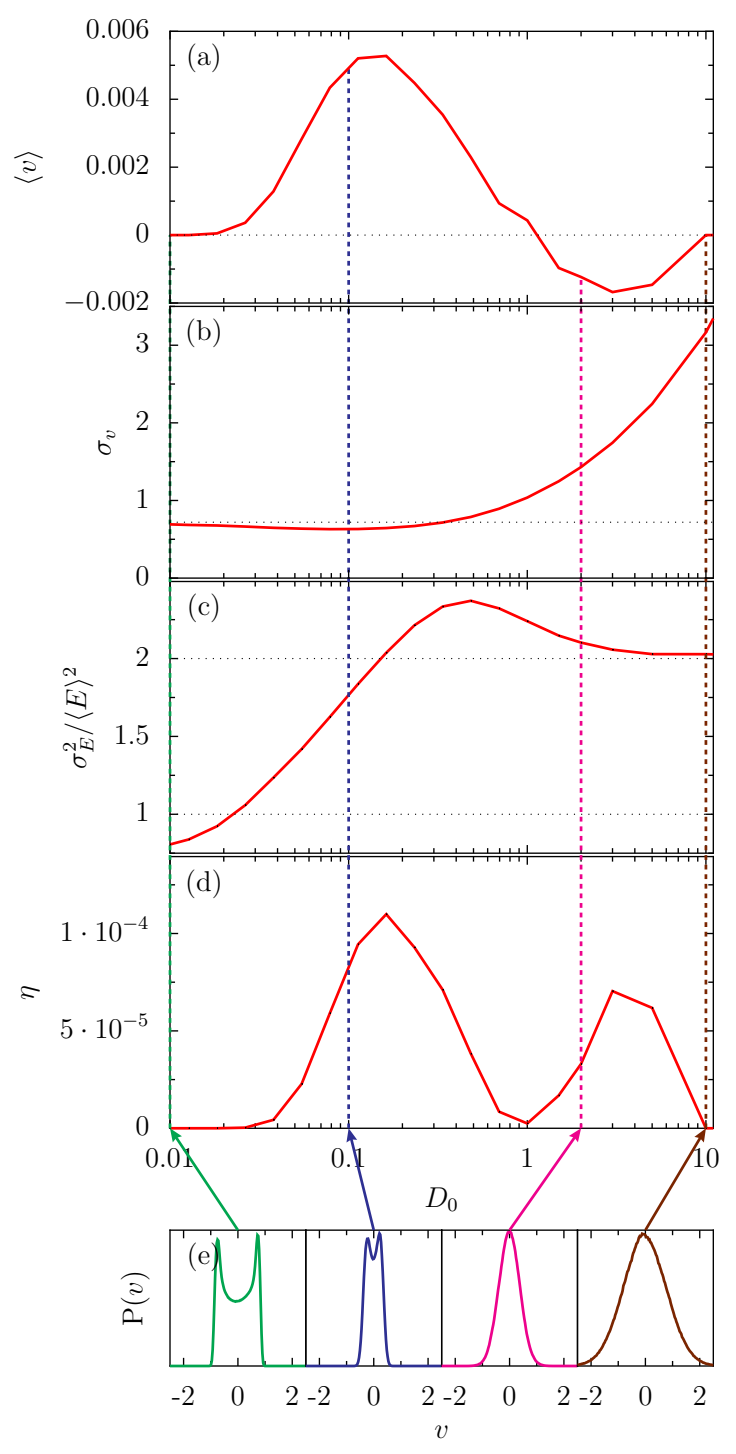

FIG. 3: (Color online) Fluctuation behavior of an inertial Brownian motor versus the noise strength $D_{0}$. (a): averaged dimensionless velocity $\langle v\rangle$; (b): the corresponding velocity variance $\sigma_{v}$; (c): fluctuations of the rescaled kinetic energy $\sigma_{E}^{2} /\langle E\rangle^{2} ;(\mathrm{d})$ : the corresponding efficiency. Numerical results obtained for the same ratchet potential as in Fig. 1 In the bottom panel (e), the velocity distribution $P(v)$ is shown for $D_{0}=0.01,0.1,2,10$. All $P(v)$ curves are normalized as in Fig. 11 The remaining rescaled parameters are: $\gamma=0.5$, $\omega=3.6$ and $a=0.8$.

slightly; nevertheless, these are still strongly suppressed in comparison to the equilibrium value 2 .

We have studied several other ratchet potentials by varying the parameters $c_{1}$ and $c_{2}$ in Eq.(4) and still found regimes where the inertial ratchet works with a high efficiency (not shown). In all these cases we found that the velocity distribution has a support concentrated mainly on one of the semi-axes. Strongly asymmetric velocity distributions are depicted with Fig. 4 (e). In contrast, with the mode $c_{2}$ set zero (see in inset in 1) we could not identify such an optimal regime for rectification of noise. The shape of these distributions just corroborates the fact that large rectification efficiencies are the result of persistent, (uni)-directional Brownian motor motion, accompanied by a strong asymmetry of the current statistics.

\section{CONCLUSION}

With this work we have elucidated, directed Brownian motor transport in rocked rachet potentials in the presence of inertia and thermal noise. We focussed on several parameter regimes and studied by numerical means the operation of this massive ratchet machine. In particular, we investigated the variation of the average current versus driving amplitude $a$ and the temperature strength $D_{0}$. Our main objective has been the behavior of the accompanying current fluctuations as a function of these transport parameters. These fluctuations crucially impact the rectification behavior, as measured by the rectification efficiency in Eq. (8).

Typically, the current values and the corresponding velocity fluctuations are so, that no appreciable rectification emerges in these inertial, rocked Brownian motors. There exist, however, tailored regimes of rachet profiles and driving parameters for which an enhancement of rectification and optimal transport do occur. These regimes are marked by a large Brownian motion transport with few back-turns only. This in turn implies a narrow, asymmetric velocity statistics with dominantly, one-sided support of either positive- or negative-valued velocities.

These novel findings for the fluctuation statistics of Brownian motor velocities can be put to use in diverse technological devices that pump and separate efficiently and reliably Brownian particles in correspondent physical [1] and biological Brownian motor systems [18]. Moreover, the results derived herein for such driven inertial Brownian motors can be applied as well to the phenomenon of Stochastic Resonance [40] in corresponding underdamped regimes.

\section{Appendix}

With this appendix we present the derivation of the expression (8) for the rectification efficiency. We combine the arguments in [5, 6, 7] and establish the efficiency $\eta$ as

$$
\eta=\frac{A}{\left|P_{i n}\right|}
$$

In the denominator, $P_{i n}$ denotes the rate of the energy input to the system. There is no overall consensus on the numerator $A$ 4, 5, 6, 7]. If $A$ denotes the rate of work done on the fluid by the Brownian motor motion, then the corresponding efficiency $\eta$ is not an appropriate measure because $A \sim\left\langle v^{2}\right\rangle$. This quantity can be relatively large 


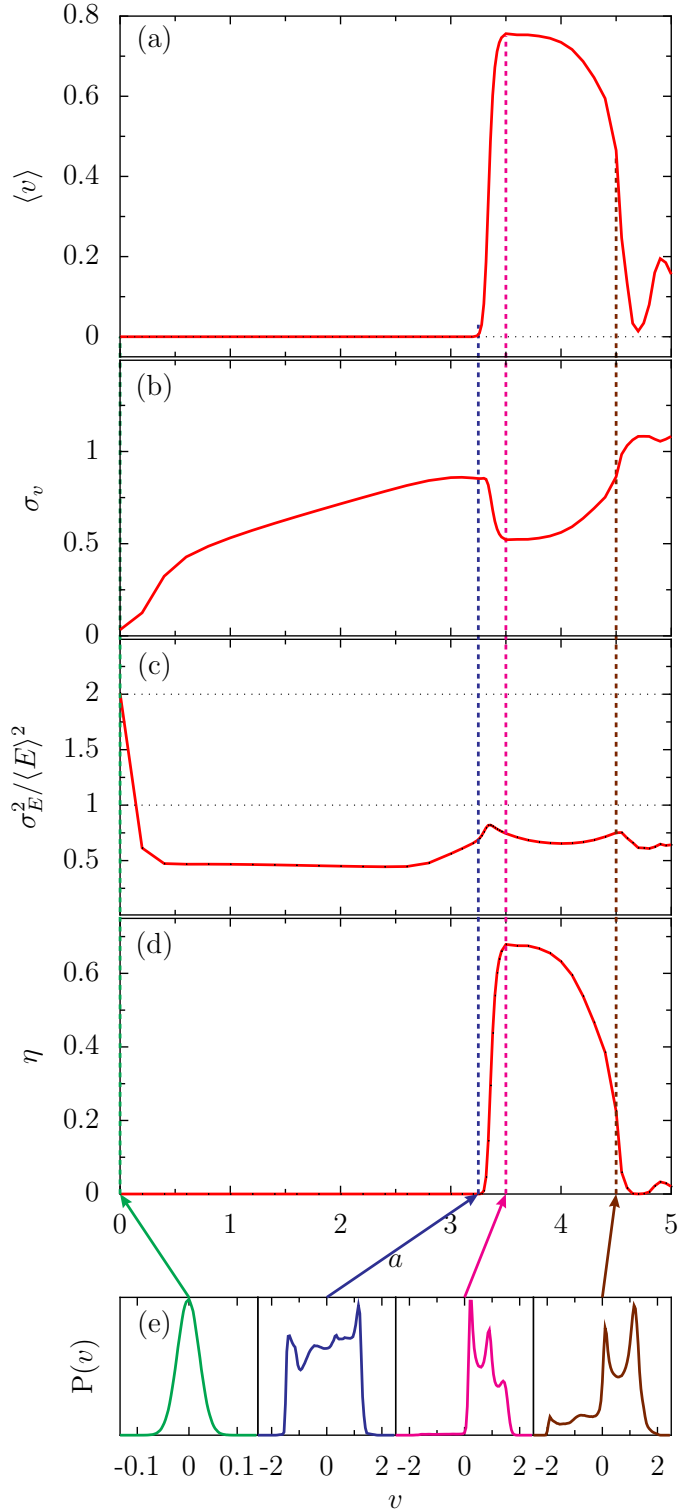

FIG. 4: (Color online) Tailoring the shape of the potential. (a): averaged dimensionless velocity $\langle v\rangle$ of the inertial Brownian motor under nonadiabatic driving conditions; (b): corresponding velocity fluctuations $\sigma_{v}$; (c): fluctuations of the rescaled kinetic energy $\sigma_{E}^{2} /\langle E\rangle^{2} ;(\mathrm{d})$ : efficiency. All quantities are plotted versus the driving amplitude $a$ for the asymmetric ratchet potential of Fig. 2 The corresponding velocity distributions $P(v)$ are shown in panel (e) for selected driving amplitudes, i.e., $a=0,3.25,3.5,4.5$. All $P(v)$ curves are normalized as in Fig. 22 The remaining parameters are: $\gamma=0.9$, $\omega=4.9$ and $D_{0}=0.001$.

even if there occurs no transport of the motor, i.e. even if $\langle v\rangle=0$ ! More suitable information on the efficiency of the transport is gained when [5, 6] $A \sim\langle v\rangle$. Following the reasoning in 5, 6, 7], we use for the output power the average friction force times the average velocity, i.e. $A=\langle\gamma v\rangle\langle v\rangle$. To calculate $P_{i n}$, let us recast (3) into the form

$$
\begin{aligned}
& d x=v d t \\
& d v=-\left(\gamma v+V^{\prime}(x, t)\right) d t+\sqrt{2 \gamma D_{0}} d W(t),
\end{aligned}
$$

where $V(x, t)=V(x)-a x \cos (\omega t)$ and $W(t)$ is the Wiener process $\left(\langle W(t)\rangle=0,\left\langle W^{2}(t)\right\rangle=t\right)$.

Now, we evaluate the ensemble and temporal averages of the re-scaled kinetic energy $G(v)=v^{2} / 2, v=v(t)$. To this aim, first we apply Itos differential calculus to the function $G(v)$ to obtain

$$
\begin{array}{r}
d\left(v^{2} / 2\right)=-\left(\gamma v^{2}+v\right. \\
\left.V^{\prime}(x, t)-\gamma D_{0}\right) d t \\
+\sqrt{2 \gamma D_{0}} v d W(t)
\end{array}
$$

The ensemble average (i.e. the average over all realization of the Wiener process denoted by $\prec \ldots \succ$ ) for the rate of change of the kinetic energy results in

$$
\begin{aligned}
\frac{d}{d t} \prec v^{2} / 2 \succ= & -\left[\gamma \prec v^{2} \succ+\prec v V^{\prime}(x) \succ\right. \\
& \left.-\prec v a \cos (\omega t) \succ-\gamma D_{0}\right],
\end{aligned}
$$

where we exploited the (Ito)-martingale property (for the part containing the Wiener process). Next, we average over the temporal period as in (5) (periodic timedependence of asymptotic probability). In doing so, we evaluate

$$
\left\langle\frac{d}{d t} v^{2}\right\rangle=\prec v^{2}(t+2 \pi / \omega) \succ-\prec v^{2}(t) \succ=0 .
$$

Likewise, for the contribution

$$
\left\langle v V^{\prime}(x)\right\rangle=\prec V(x(t+2 \pi / \omega)) \succ-\prec V(x(t)) \succ=0 .
$$

Consequently, we obtain

$$
0=-\gamma\left[\left\langle v^{2}\right\rangle-D_{0}\right]+P_{i n}
$$

where the combined average $\left.P_{i n}=\langle v(t) a \cos \omega t)\right\rangle$ is the input energy to the system per unit time. Thus, upon combining (10) and (17) the relation in (8) emerges. We also emphasize here, that our scheme for the efficiency of rectification at zero bias is independent of the transport friction-coefficient $\gamma$. This feature is in agreement with the corresponding result by Suzuki and Munakata [7].

\section{Acknowledgment}

The authors gratefully acknowledge financial support by the DAAD-KBN (German-Polish project Stochastic Complexity) (PH and JE), the Foundation for Polish Science [Fundacja na Rzecz Nauki Polskiej] (PH), the ESF (Program Stochastic Dynamics), the Deutsche Forschungsgemeinschaft via grant HA 1517/13-4, the Graduiertenkolleg 386 (P.H.,P.T.) and the collaborative research grant SFB 486. 
[1] for a review see P. Hänggi and R. Bartussek, Lect. Notes Phys. 476, 294 (1996); R. D. Astumain, Science, 276, 917 (1997); R.D. Astumian and P. Hänggi, Physics Today 55 (No.11), 33 (2002); P. Reimann and P. Hänggi, Appl. Phys. A 75, 169 (2002); P. Reimann, Phys. Rep. 361, 57 (2002); H. Linke, Appl. Phys. A 75, 167 (2002); a special issue on Brownian motors.

[2] P. Jung, J. G. Kissner, and P. Hänggi, Phys. Rev. Lett. 76. 3436 (1996).

[3] N. SanchezSalas and A. C. Hernandez, Phys. Rev. E 68, 046125 (2003).

[4] H. X. Zhou and Y. D. Chen, Phys. Rev. Lett. 77, 194 (1996); K. Sekimoto, J. Phys. Soc. Jpn. 66, 1234 (1997); H. Kamegawa, T. Hondou, and F. Takagi, Phys. Rev. Lett. 80, 5251 (1998); I. M. Sokolov and A. Blumen, Chem. Phys. 235, 39 (1998); J. M. R. Parrondo, J. M. Blanco, F. J. Cao, and R. Brito, Europhys. Lett. 43, 248 (1998); A. Parmeggiani, F. Jülicher, A. Ajdari, and J. Prost, Phys. Rev. E 60, 2127 (1999); K. Sekimoto, F. Takagi and T. Hondou, Phys. Rev. E 627759 (2000); M. Asfaw and M. Bekele, Eur. Phys. J. B 38, 457 (2004).

[5] I. Derenyi, M. Bier, and R.D. Astumian, Phys. Rev. Lett. 83, 903 (1999);

[6] H. Wang and G. Oster, Europhys. Lett. 57, 134 (2002).

[7] D. Suzuki and T. Munakata, Phys. Rev. E 68, 021906 (2003).

[8] J. M. R. Parrondo and B. J. Cisneros, Appl. Phys. A 75, 179 (2002).

[9] M. Schreier, P. Reimann, P. Hänggi, and E. Pollak, Europhys. Lett. 44, 416 (1998).

[10] C. Costantini and F. Marchesoni, Europhys. Lett. 48, 491 (1999).

[11] J. A. Freund and L. Schimansky-Geier, Phys. Rev. E 60, 1304 (1999); B. Lindner and L. Schimansky-Geier, Phys. Rev. Lett. 89, 230602 (2002).

[12] J. W. Shuai and P. Jung, Fluct. Noise Lett. 2, L139 (2003).

[13] A. B. Kolomeisky and M. E. Fisher, Biophys. J. 84, 1642 (2003); M. E. Fisher and A. B. Kolomeisky, Physica A 274, 241 (1999).

[14] P. Reimann, C. Van den Broeck, H. Linke, P. Hänggi, J. M. Rubi, and A. Pérez-Madrid, Phys. Rev. Lett. 87, 010602 (2001).

[15] P. Reimann, C. Van den Broeck, H. Linke, P. Hänggi, J. M. Rubi, and A. Pérez-Madrid Phys. Rev. E 65, 031104 (2002).

[16] B. Lindner, M. Kostur, and L. Schimansky-Geier, Fluct. Noise Lett. 1, R25 (2001).

[17] M. Bier, Phys. Rev. Lett. 91, 148104 (2003).

[18] F. Jülicher, A. Ajdari, and J. Prost, Rev. Mod. Phys. 69,
1269 (1997); R. D. Astumian, Appl. Phys. A 75, 193 (2002); J. Howard, Mechanics of Motor Proteins and the Cytoskeleton (Sinauer Ass., Sunderland, 2001).

[19] B. Lindner et al., Phys. Rev. E 59, 1417 (1999).

[20] J. L. Mateos, Phys. Rev. Lett. 84, 258 (2000); J. L. Mateos, Physica A 325, 92 (2003).

[21] S. Flach, O. Yevtushenko, and Y. Zolotaryuk, Phys. Rev. Lett. 84, 2358 (2000); S. Denisov and S. Flach, Phys. Rev. E 64, 056236 (2001); H. Schanz, M. F. Otto, R. Ketzmerick, and T. Dittrich, Phys. Rev. Lett. 87, 070601 (2001); T. Dittrich, R. Ketzmerick, M. F. Otto, and H. Schanz, Ann. Physik (Leipzig) 9, 755 (2000).

[22] M. Barbi and M. Salerno, Phys. Rev. E 62,1988 (2000).

[23] W. S. Son, I. Kim, Y. J. Park, and C. M. Kim, Phys. Rev. E 68, 067201 (2003).

[24] M. Borromeo, G. Costantini and F. Marchesoni, Phys. Rev. E 65, 041110 (2002).

[25] H. A. Larrondo, F. Family and C. M. Arizmendi, Physica A 303, 67 (2002); H. A. Larrondo, C. M. Arizmendi and F. Family, Physica A 320, 119 (2003).

[26] T. Sintes and K. Sumithra, Physica A 312, 86 (2002).

[27] S. Sengupta, R. Guantes, S. Miret-Artes, and P. Hänggi, Physica A 338, 406 (2004).

[28] D. Fleishman, A. E. Filippov, and M. Urbakh, Phys. Rev. E 69, 011908 (2004).

[29] E. Pollak, J. Bader, B. J. Berne, and P. Talkner Phys. Rev. Lett. 70, 3299 (1993); M. Borromeo and F. Marchesoni, Phys. Rev. Lett. 84, 203 (2000).

[30] E. Ganz, S. K. Theiss, I.-S. Hwang, and J. Golovchenko, Phys. Rev. Lett. 68, 1567 (1992).

[31] D. C. Senft and G. Ehrlich, Phys. Rev. Lett. 74, 294 (1995).

[32] J. Ellis and J. P. Toennies, Phys. Rev. Lett. 70, 2118 (1993).

[33] R. Bartussek, P. Hänggi, and J. G. Kissner, Europhys. Lett. 28, 459 (1994).

[34] I. Zapata et al., Phys. Rev. Lett. 77, 2292 (1996).

[35] J. Luczka, R. Bartussek, and P. Hänggi, Europhys. Lett 31, 431 (1995); R. Bartussek, P. Reimann, and P. Hänggi, Phys. Rev. Lett. 76, 1166 (1996).

[36] S. Savelev, F. Marchesoni, P. Hänggi, and F. Nori, Europhys. Lett. 67, 179 (2004).

[37] E. Heinsalu, R. Tammelo, and T. Ord, Phys. Rev. E 69, 021111 (2004).

[38] P. Jung and P. Hänggi, Phys. Rev. A 41, 2977 (1990); P. Jung, Phys. Rep. 234, 175 (1993).

[39] P. Hänggi and H. Thomas, Phys. Rep. 88, 207 (1982).

[40] L. Gammaitoni, P. Hänggi, P. Jung, and F. Marchesoni, Rev. Mod. Phys. 70, 223 (1998). 